\title{
Improving Energy Expenditure Estimation for Physical Activity
}

\author{
KUAN ZHANG, F. XAVIER PI-SUNYER, and CAROL N. BOOZER \\ NY Obesity Research Center, St. Luke's-Roosevelt Hospital and Institute of Human Nutrition, Department of Medicine, \\ Columbia University, New York, NY
}

\begin{abstract}
ZHANG, K., F. X. PI-SUNYER, and C. N. BOOZER. Improving Energy Expenditure Estimation for Physical Activity. Med. Sci. Sports Exerc., Vol. 36, No. 5, pp. 000-000, 2004. Purpose: The purpose of this study was to validate the Intelligent Device for Energy Expenditure and Activity (IDEEA) for estimation of energy expenditure during a variety of activities. An additional aim was to improve the accuracy of the estimation of energy expenditure of physical activity based on second-by-second information of type, onset, and duration of activity. Methods: This study included two tests: a mask calorimetry test with 27 subjects [age $=33.7 \pm 13.8$ (mean \pm $\left.\mathrm{SD}) \mathrm{yr} ; \mathrm{BMI}=24.8 \pm 4.8 \mathrm{~kg} \cdot \mathrm{m}^{-2}\right]$ and a respiratory chamber calorimetry test with 10 subjects $(\mathrm{age}=32.9 \pm 12.4 \mathrm{yr} ; \mathrm{BMI}=26.1$ $\pm 5.6 \mathrm{~kg} \cdot \mathrm{m}^{-2}$ ). In the mask test, the subjects performed activities (sitting, standing, lying down, level treadmill walking, and running at different speeds) for 50-min durations. For the chamber test, subjects lived in the metabolic chamber for $23 \mathrm{~h}$ and performed three exercise sessions to compensate for the confined environment. Results: The results showed significant correlations $(P<0.0001)$ between energy expenditure estimated by IDEEA and energy expenditure measured by the calorimeters with an accuracy $>95 \%$. After corrections for the decrease in sleeping metabolic rate, the estimation accuracy for the chamber test was increased by 1-96.2\%, whereas the estimation accuracy for nighttime activity was significantly improved by $4-99 \%$. Conclusion: IDEEA provides a suitable method for estimating the energy expenditure of physical activity. It provides both instantaneous and cumulative estimates of energy expenditure over a given period. Key Words: FREE-LIVING, ENERGY EXPENDITURE OF PHYSICAL ACTIVITY, INDIRECT CALORIMETRY, IDEEA, METABOLIC RATE
\end{abstract}

$\mathrm{D}$ etermination of the amount of physical activity (PA) needed to derive specific health benefits requires accurate and reliable methods of measuring energy expenditure (EE) in free-living individuals $(4,5,14,21)$. However, because of the complexities of human PA and diversified life styles, it has been virtually impossible to accurately record free-living PA/exercise and the correspondent EE of activity on a daily basis. The measurement of EE in free-living individuals always represents a methodological challenge. The methods used to measure EE in free-living conditions include doubly labeled water, heart rate monitors, pedometers, accelerometers, diet records, and activity questionnaires. Unfortunately, as Luke et al. (14) pointed out, the accurate measurement of PA and EE is problematic, and all of the existing measurement techniques have significant limitations. Doubly labeled water $(3,16,20,23,25)$ estimates $\mathrm{EE}$ by measuring $\mathrm{CO}_{2}$ output in the free-living state and has been frequently used, but it is

\footnotetext{
Address for correspondence: Kuan Zhang, Ph.D., NY Obesity Research Center, St. Luke's-Roosevelt Hospital Center, 1111 Amsterdam Avenue, New York, NY 10025; E-mail: kz6@ columbia.edu.

Submitted for publication July 2003.

Accepted for publication December 2003.
}

0195-9131/04/3605-0001

MEDICINE \& SCIENCE IN SPORTS \& EXERCISE E

Copyright (C) 2004 by the American College of Sports Medicine

DOI: 10.1249/01.MSS.0000126585.40962.22 expensive and takes at least $3 \mathrm{~d}$ to get only average daily EE without detection of type, onset, duration, and intensity of components of PA and their energy cost. Heart rate monitors $(8,14,15)$, pedometers $(8,9,27)$, and accelerometers $(3,5-$ $7,10,12,16,17,25)$ give poor and inconsistent results in estimating individual energy expenditure of physical activity (EEpa). Diet records $(22,24,28)$ and activity questionnaires $(1,18,26)$ are less reliable.

Recently, a portable device, Intelligent Device for Energy Expenditure and Activity (IDEEA, MiniSun, CA) has been developed that shows promise in the estimation of EEpa. Major functions of the IDEEA include: 1) recording body motion and posture changes second by second on 24-h basis; 2) identifying all major PA and posture types of daily life; 3) providing accurate recordings of the onset, duration, and frequency of the activities and performing detailed analysis of category and distribution of these activities; and 4) computing the amount and intensity (e.g., the speed of walking or running).

Conceptually, the use of IDEEA offers an ideal solution because it directly measures type, onset, duration, intensity, and frequency of PA, capturing the fundamental nature of PA. Our previous study (30) demonstrated that IDEEA could accurately detect the type, onset, duration, and intensity of various daily PA with an accuracy of more than $98 \%$. IDEEA also accurately predicted the speed of gaits (walking and running), which is a predominant factor relating to EEpa, with a pooled correlation between predicted and actual speeds of walking and running of $0.986(P \leq 0.0001)$ 
TABLE 1. Subject characteristics for the mask test (mean \pm SD) (range).

\begin{tabular}{lc}
\hline No. of subjects & $\mathbf{2 7}$ \\
\hline Age $(\mathrm{yr})$ & $\mathbf{( 1 0}$ males and $\mathbf{1 7}$ females $)$ \\
Body weight $(\mathrm{kg})$ & $33.7 \pm 13.8(15 \sim 61)$ \\
Height $(\mathrm{cm})$ & $69.1 \pm 14.1(52.6 \sim 103.4)$ \\
BMl $\left(\mathrm{kg} \cdot \mathrm{m}^{-2}\right)$ & $167.0 \pm 7.9(154.0 \sim 190.0)$ \\
\hline
\end{tabular}

(30). This makes it possible to accurately estimate EEpa without interrupting a subject's daily PA.

The purpose of this study was to examine whether IDEEA could provide a valid estimate of EE during a variety of activities compared with indirect calorimetry. A further aim was to improve the accuracy of estimation of EEpa based on second-by-second information of type, onset, and duration of activity collected by IDEEA.

\section{METHODS}

Subjects. Subjects were recruited through the hospital and neighborhood community by phone calls and e-mail. The subjects received a small monetary compensation for their participation. A total of 30 subjects (11 males and 19 females) were included in the study. Among them, 27 (10 males and 17 females) participated in the mask calorimeter test while 10 (five males and five females) participated in the respiratory chamber test. The characteristics of all subjects are shown in Tables 1 and 2. All subjects were free of any impairment of the locomotor system.

The study was approved by the Institutional Review Board at St. Luke's-Roosevelt Hospital Center, New York, NY. All subjects signed written consent to participate. The consent forms for subjects under age of 18 were signed by their parents and those subjects were accompanied by their parents during the test.

Energy expenditure. Energy expenditure was estimated by IDEEA, a device described elsewhere in detail (30). Briefly, IDEEA consists of one recorder and five small sensors that are placed on the chest, on the frontal part of each thigh, and under each foot, which measure the acceleration and angle of each body segment. The sensors are connected to the recorder by thin and flexible wires $(2 \mathrm{~mm}$ outer diameter). Before the test, sensors are applied to body locations, and the IDEEA recorder connected to a personal computer by a communication cable. A windows-based interface program controls the communication between IDEEA and a laptop or desktop computer (PC). After entering filename, weight, height, age, gender, an optional study ID, and short memo from the keyboard of the PC, the cable can be detached and the IDEEA started to begin to

TABLE 2. Subject characteristics for the chamber test (mean \pm SD) (range).

\begin{tabular}{lc}
\hline No. of subjects & $\mathbf{1 0}$ \\
Age $(\mathrm{yr})$ & $\mathbf{5}$ males and $\mathbf{5}$ females $)$ \\
Body weight $(\mathrm{kg})$ & $32.9 \pm 12.4(20 \sim 53)$ \\
Height $(\mathrm{cm})$ & $75.8 \pm 16.6(54.0 \sim 103.4)$ \\
BMI $\left(\mathrm{kg} \cdot \mathrm{m}^{-2}\right)$ & $170.6 \pm 10.0(157.0 \sim 190.0)$ \\
\hline
\end{tabular}

TABLE 3. The protocol for the mask test.

\begin{tabular}{lccccc}
\multicolumn{1}{c}{ Activity } & $\begin{array}{c}\text { Group } \\
\mathbf{1}\end{array}$ & $\begin{array}{c}\text { Group } \\
\mathbf{2}\end{array}$ & $\begin{array}{c}\text { Group } \\
\mathbf{3}\end{array}$ & $\begin{array}{c}\text { Group } \\
\mathbf{4}\end{array}$ & $\begin{array}{c}\text { Duration } \\
\text { (min) }\end{array}$ \\
\hline Sitting & & & & & 4 \\
Standing & & & & & 4 \\
Lying down & & & & & 4 \\
Walking (mph) & 1.8 & 1.8 & 1.8 & 1.8 & 5 \\
Walking (mph) & 4 & 3.7 & 3.1 & 2.4 & 3 \\
Running (or walking) (mph) & 6.5 & 5.6 & 4.8 & 3 & 3 \\
Walking (mph) & 3 & 2.5 & 2 & 2 & 5 \\
Running (or walking) (mph) & 6 & 5 & 4.5 & 2.5 & 3 \\
Walking (mph) & 3.5 & 3 & 2.5 & 2.3 & 5 \\
Running (or walking) (mph) & 5.5 & 4.5 & 3.8 & 2.8 & 3 \\
Walking (mph) & 2.2 & 2.2 & 2.2 & 2.2 & 5 \\
\hline
\end{tabular}

collect data. The sensors are attached by a porous, hypoallergic medical tape during the measurement and have to be taken off during bathing. At the end of data collection, the subject can power off IDEEA. Data will remain in IDEEA until they are loaded to a computer. The ability of IDEEA to correctly identify type of activities and to quantify PA intensity allows calculation of EE in free-living conditions. This is accomplished by use of multiple equations for EE of activities (such as sit, stand, walk and run at different speeds). The EE of activities were obtained by instructing subjects to perform these activities and recording their EE using whole-room calorimeter or metabolic carts. Some of the equations were obtained from well-researched databases for EE costs of specific activities (2). Resting energy expenditure (REE) and sleeping energy expenditure (SEE) were calculated according to demographic information. The models of RMR and SMR were derived from approximately 500 subjects who spent $23 \mathrm{~h}$ in metabolic chambers performing known activities, such as sleep, sit, and REE test. These equations were then built into the IDEEA System. The IDEEA System identifies the type of on-going activity and calculates its intensity such as the speed of walking or the rate of standing up, and uses the correct equation for the calculation of EE during that moment. This process continues for each second so that a dynamic EE curve is obtained at the end of recording. After data are downloaded and processed, results such as EE and speeds of walking and running are analyzed by ActView, a Window-based program that provides detailed information including type, onset, duration, intensity, and EE of each PA.

To validate IDEEA estimated EE of PA, we measured EE of subjects wearing IDEEA by using a nonportable mask calorimeter and a respiratory chamber calorimeter (open-air

TABLE 4. The protocol for the chamber test.

\begin{tabular}{ll}
\hline \multicolumn{1}{c}{ Time } & \multicolumn{1}{c}{ Activity } \\
\hline 8:00 a.m. & Arrive at the chamber \\
9:00 a.m. & Enter the chamber \\
9:30 a.m. & Breakfast \\
11:00 a.m. & Exercise 1 on treadmill (walking for $15 \mathrm{~min}$ ) \\
12:30 p.m. & Lunch \\
3:00 p.m. & Exercise 2 on treadmill (running for $10 \mathrm{~min}$ or walking for $15 \mathrm{~min}$ ) \\
5:30 p.m. & Dinner \\
7:00 p.m. & Exercise 3 on treadmill (walking for $15 \mathrm{~min}$ ) \\
11:00 a.m. & Go to sleep \\
8:00 a.m. & Exit chamber \\
\hline
\end{tabular}


TABLE 5. Accuracy of IDEEA estimation of EE for the mask test.

\begin{tabular}{cccccc}
\hline & All Subjects & Male Subjects & Female Subjects & BMI $<25$ & BMI $\geq 25$ \\
\hline Accuracy & $98.9 \pm 6.0 \%$ & $95.8 \pm 2.9 \%$ & $100.7 \pm 6.7 \%$ & $98.1 \pm 6.1 \%$ & $99.2 \pm 6.0 \%$ \\
& $(90.3 \sim 110.7 \%)$ & $(92.1 \sim 100.0 \%)$ & $(90.3 \sim 110.7 \%)$ & $(90.3 \sim 108.3 \%)$ & $(92.9 \sim 110.7 \%)$ \\
\hline
\end{tabular}

circuits). The mask calorimeter is used for short and fast response measurement in which a mask (Hans Rudolph, Kansas City, MO) is connected to a flow meter that measures the flow rate through a tube. The airflow was purged by a fan to generate a significantly higher flow than pulmonary ventilation and a slightly negative pressure to prevent leak. The chamber calorimeter, as described previously (29), is an airtight room (22,000-L volume) equipped with a bed, chair, desk, television, VCR, radio, telephone, bicycle, sink, and toilet. The temperature of the room is maintained at $23^{\circ} \mathrm{C} \pm 0.2^{\circ} \mathrm{C}$. A fan draws mixed-air (sample air) out of the chamber through a flow meter, while fresh air (reference air) is forced into the chamber by the resulting negative pressure.

For both calorimeters, the air samples are dried and wellmixed before sending to oxygen analyzer (Magnos 4G, Hartman \& Braun) and carbon dioxide analyzer (Uras 3G, Hartman \& Braun). The analyzers were calibrated using reference air before the tests. During the tests, the flow was measured by a precision mass flowmeter (error $\pm 0.5 \%$, TeleDyne), and the differences of $\mathrm{O}_{2}$ and $\mathrm{CO}_{2}$ concentrations between expired air and reference air are measured by analyzers; therefore, the $\mathrm{O}_{2}$ consumption and $\mathrm{CO}_{2}$ production could be determined to calculate $\mathrm{EE}$. The results are corrected for barometric pressure, flow rate, and humidity.

Experimental protocols. The ability of IDEEA to estimate EEpa was tested by two protocols: a mask calorimeter test protocol and a chamber calorimeter test protocol. For the mask test, subjects wore masks with a Y-shape valve and sealing gel placed on the edge of masks (Hans Rudolph, Kansas City, MO) to prevent air leaking. After the placement of IDEEA, subjects were then asked to sit, stand and lie down for $4 \mathrm{~min}$, respectively, before mounting a treadmill (Trimline 4650, Hebb Industries, Inc, Tyler, TX) to perform the walking and running tests on a level treadmill at different speeds according to the protocol described in Table 3 . The complete test took about $50 \mathrm{~min}$, including all transitions between activities.

For the chamber test, subjects were required to stay $23 \mathrm{~h}$ in the respiratory chamber. After an overnight fast, subjects came to the hospital at 8:00 am to have resting metabolic rate (RMR) measured. The food intake for the 23-h chamber stay was estimated by $1.5 \times$ REE, and regular hospital food was supplied (30\% fat, $23 \%$ protein, and $47 \%$ carbohydrate) in three meals. After placement of the IDEEA device, subjects entered the chamber at 9:00 am. During the chamber stay, subjects exercised three times including two times walking and one time running or three times walking only, depending on the capability of the subject (Table 4). In order to encourage more activity, subjects were asked to not lie down before 9:00 p.m. except for a 1-h break after lunch.

Data analysis. EE is described by metabolic rate (MR) in kilocalories per minute for data analysis. After each test, the data from IDEEA was downloaded and processed on a personal computer. MR measured by indirect calorimetry and MR estimated by the IDEEA were analyzed across $30 \mathrm{~s}$ and 2 min intervals for the mask test and chamber test, respectively. The accuracy was used to compare the estimate of EE by IDEEA with calorimetry measurement. We define the accuracy in this study as: accuracy $=[($ mean MR from IDEEA)/(mean MR from calorimeter) $]^{*} 100 \%$. The mean MR from IDEEA and calorimetry were calculated for the entire test period, including all transitions between activities. This may have reduced the accuracy of IDEEA but is closer to a real-life situation. Correlations between EE estimated by IDEEA and EE measured by indirect calorimeter were computed. The intraclass correlation (ICC) was used to compare accuracy with subject characteristics (age, gender, and BMI). Basic descriptive statistics by overall, gender and BMI were calculated (Tables 5-8). Bland-Altman plots were to determine whether bias is statistically significant. These analyses were performed using SPSS 10.0. Statistical, significance was defined at $P<0.05$.

\section{RESULTS}

The mask test. Figure 1 shows a representative profile of MR estimated by IDEEA and measured by mask calorimeter with recording intervals of $30 \mathrm{~s}$. Accuracies of IDEEA estimation are given in Table 5. For 27 subjects who participated in the mask test, the overall accuracy was 98.9 $\pm 6.0 \%$ (mean $\pm \mathrm{SD}$, range: $90.3 \% \sim 110.7 \%$ ). The average accuracy for male subjects $(95.8 \pm 2.9 \%, 92.1 \sim$ $100.0 \%)$ was lower than accuracy for female subjects (100.7 $\pm 6.7 \%, 90.3 \sim 110.7 \%)$. Accuracy for subjects with a BMI $<25(98.1 \pm 6.1 \%, 90.3 \sim 108.3 \%)$ was close to the accuracy for subjects with a BMI $\geq 25$ (99.2 $\pm 6.0 \%, 92.9$ $\sim 110.7 \%$ ). ICC shown in Table 6 compares accuracy with subject characteristics (gender, age, BMI, weight, and height). It shows that the accuracy was not statistically significantly affected by age $(P=0.858)$, BMI $(P=0.847)$,

TABLE 6. ICC between accuracy and age, BMI, weight, and height for the mask test.

\begin{tabular}{cccccc}
\hline & \multicolumn{5}{c}{ Correlation } \\
\cline { 2 - 5 } & Gender & Age & BMI & Weight & Height \\
\hline Accuracy & 0.408 & -0.039 & -0.040 & -0.146 & -0.240 \\
& $(P=0.038)$ & $(P=0.858)$ & $(P=0.847)$ & $(P=0.477)$ & $(P=0.238)$ \\
\hline
\end{tabular}


TABLE 7. Accuracy of IDEEA estimation of EE for the chamber test.

\begin{tabular}{|c|c|c|c|c|c|c|c|}
\hline & $\begin{array}{c}\text { All } \\
\text { Subjects }\end{array}$ & $\begin{array}{l}\text { Male } \\
\text { Subjects }\end{array}$ & $\begin{array}{l}\text { Female } \\
\text { Subjects }\end{array}$ & BMI $<25$ & $B M I \geq 25$ & Day Time & Night Time \\
\hline Accuracy & $\begin{array}{c}95.2 \pm 2.3 \% \\
(91.5 \sim 98.5 \%)\end{array}$ & $\begin{array}{c}94.2 \pm 2.0 \% \\
(91.5 \sim 96.2 \%)\end{array}$ & $\begin{array}{c}96.1 \pm 2.4 \% \\
(92.8 \sim 98.5 \%)\end{array}$ & $\begin{array}{c}96.8 \pm 2.1 \% \\
(92.8 \sim 98.5 \%)\end{array}$ & $\begin{array}{c}94.2 \pm 2.4 \% \\
(91.5 \sim 98.0 \%)\end{array}$ & $\begin{array}{c}95.0 \pm 3.1 \% \\
(91.6 \sim 101.0 \%)\end{array}$ & $\begin{array}{c}95.3 \pm 2.7 \% \\
(91.0 \sim 98.4 \%)\end{array}$ \\
\hline
\end{tabular}

weight $(P=0.477)$, and height $(0.238)$ but was significantly affected by gender $(P=0.038)$.

A highly significant correlation was found $(\mathrm{r}=0.973, P$ $<0.0001$ ) between MR estimated by IDEEA and MR measured by mask calorimeter (Fig. 2). The Bland-Altman bias plot for the mask test is shown in Figure 3. Correlation $\mathrm{r}=0.1828(P=0.7702)$ for the difference of IDEEA and the mask against the average of two methods indicates the difference of two methods is not significantly correlates to the average of two methods. The bias -0.075 within $95 \%$ confidence interval (CI) of -0.181 to 0.031 , lower $95 \%$ limit of agreement -0.599 with $95 \%$ CI of -0.772 to -0.427 , and upper $95 \%$ limit of agreement is 0.449 with 95\% CI of 0.276-0.621 demonstrates that the degree of agreement is acceptable for the mask test.

The chamber test. Figure 4 shows a representative profile in 2-min intervals of MR estimated by IDEEA and measured by chamber calorimeter during the test. The results from 10 subjects who participated in the chamber test also show high correlation between MR from IDEEA and MR from the chamber calorimeter $(\mathrm{r}=0.959, P<0.0001)$ (Fig. 5).

The overall accuracy was $95.1 \pm 2.3 \%$ (mean \pm SD, range: 91.5-98.5\%) (Table 6). The accuracy for female subjects $(96.1 \pm 2.4 \%$, range: $92.8-98.5 \%)$ was slightly higher than the accuracy for male subjects $(94.2 \pm 2.0 \%$, range: $91.5-96.2 \%$ ), but these were not significantly different $(\mathrm{r}=0.432, P=0.213)$. There was no significant difference between the accuracy for subjects with a BMI $<$ $25(96.8 \pm 2.1 \%$, range: $92.8-98.5 \%)$ and for subjects with a BMI $\geq 25$ (94.2 $\pm 2.4 \%$, range: $91.5-98.0 \%, r=-0.04$, $P=0.847)$. There was no difference between accuracies during daytime $(95.0 \pm 3.1 \%$, range: $91.6-101.0 \%)$ and nighttime (95.3 $\pm 2.7 \%$, range: $91.0-98.4 \%$ ), which shows consistent estimation for dynamic and static activities. There was also no statistically significant effect of weight $(\mathrm{r}=$ $-0.146, P=0.160)$ and height $(\mathrm{r}=-0.240, P=0.813)$ (Table 7).

The Bland-Altman bias plot for the chamber test is shown in Figure 6. Correlation $\mathrm{r}=0.0663(P=0.8464)$ for the difference of IDEEA and the chamber against the average of the two methods indicates the difference of two methods is not significantly correlates to the average of two methods.
The bias is -0.057 with $95 \% \mathrm{CI}$ of -0.122 to 0.007 . Lower $95 \%$ limits of agreement is -0.233 with $95 \%$ CI of -0.328 to -0.138 and upper $95 \%$ limits of agreement is 0.118 with $95 \%$ CI $0.023-0.213$. These analyses show that bias is not statistically significant for the chamber test.

\section{DISCUSSION}

The most variable component of daily EE is the thermic effect of PA, which can change significantly for an individual from day to day $(11,20)$. In very sedentary individuals, EEpa can be less than $100 \mathrm{kcal} \cdot \mathrm{d}^{-1}$, whereas in endurancetrained athletes it may exceed $3000 \mathrm{kcal} \cdot \mathrm{d}^{-1}$. Thus, accurate assessment of EEpa is critical to understanding the effect of PA on energy balance.

The present study was conducted to determine if IDEEA can accurately estimate EEpa compared with indirect calorimetry in two tests of 50-min and 23-h periods, respectively. Results show significant correlations with average accuracy of more than $95 \%$. For the two-tailed test with significance criterion $P<0.05$, the power for the chamber test ( $\mathrm{r}=0.959$, sample size $N=10$ ) is 0.98 , and the power for the mask test $(\mathrm{r}=0.973$, sample size $N=27)$ is approaching $1.00(\geq 0.99)$, which means the numbers of subjects for both tests were adequate. Another interesting point can be found in Table 8, where the correlations between accuracy and both gender and weight are greater than 0.408 , but they are not significantly correlated $(P=0.213$ and $P=0.160$, respectively) for the chamber test. The reason for this is probably due to the relatively small sample size of the chamber test (10 subjects) compared with mask test (27 subjects) although power analysis shows the sample sizes for both tests were acceptable.

Although the overall accuracy of estimation is satisfying for both mask test $(98.9 \%)$ and chamber test $(95.2 \%)$, IDEEA underestimated EE for certain subjects and overestimated EE for others up to $10 \%$ for the worst case. There are a few possible reasons that could cause this variation. First, the level walking and running test were done on a treadmill. Pugh (19) showed that $7.5 \%$ of total energy cost is used to overcome air resistance for middle-distance races and $13 \%$ in a sprint. Subjects who are very familiar with the treadmill would use less energy on the treadmill than on the

TABLE 8. ICC between accuracy and age, BMI, weight, and height for the chamber test.

\begin{tabular}{cccccc}
\hline & \multicolumn{4}{c}{ Correlation } \\
\cline { 2 - 5 } & Gender & Age & BMI & Weight & Height \\
\hline Accuracy & 0.432 & 0.129 & -0.387 & -0.481 & -0.086 \\
& $(P=0.213)$ & $(P=0.722)$ & $(P=0.269)$ & $(P=0.160)$ & $(P=0.813)$ \\
\hline
\end{tabular}




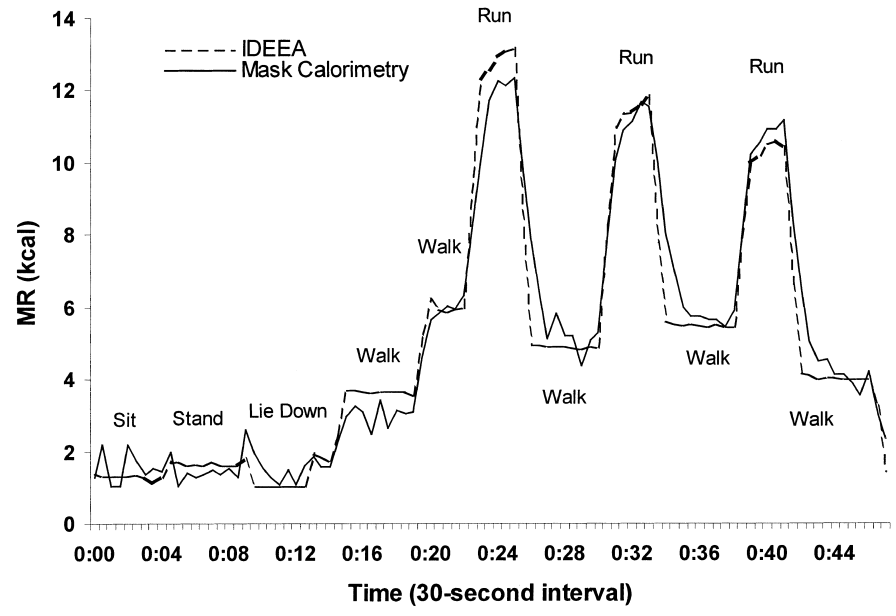

FIGURE 1-Representative profiles of MR estimated by IDEEA and MR measured by the mask calorimeter.

ground, thus IDEEA could overestimate EE (from our observation, all subjects with accuracies more than $106 \%$ were very good runners on the treadmill). For subjects who were not familiar with the treadmill, the compensatory movements would increase $\dot{\mathrm{VO}}_{2}$ and $\mathrm{EE}$ as compared on the ground at the same speed. In this case, IDEEA could underestimate EE (in this study, the subject with the lowest accuracy $90.3 \%$ was a first time user of a treadmill). This could be solved either by field walking and running (need a portable calorimetry) or by increasing the inclination of treadmill to mimic the air resistance. Second, the overestimation of $\mathrm{EE}$ in individuals might be attributed to their enhanced fitness level (12). Unfortunately, fitness was not measured in this study. Those limitations can be overcome by using doubly labeled water in a future study.

Third, PA involving arm movement could not be identified by IDEEA, which could result in underestimation of EE. Fourth, EE during the transition between two physical activities could not be well estimated because of a lack of a

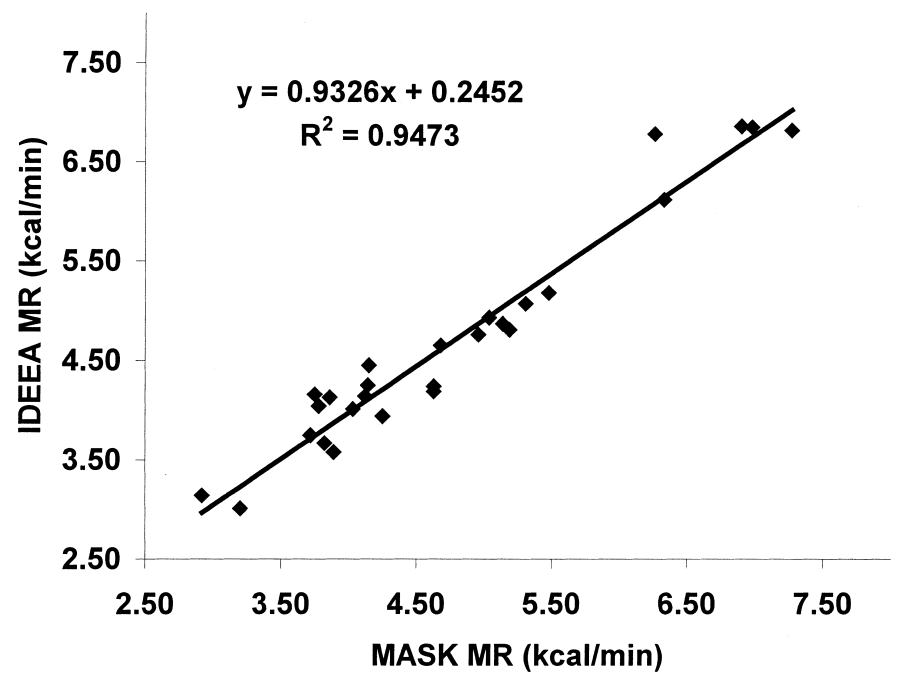

FIGURE 2-Comparison between MR from IDEEA and MR from the mask calorimeter.

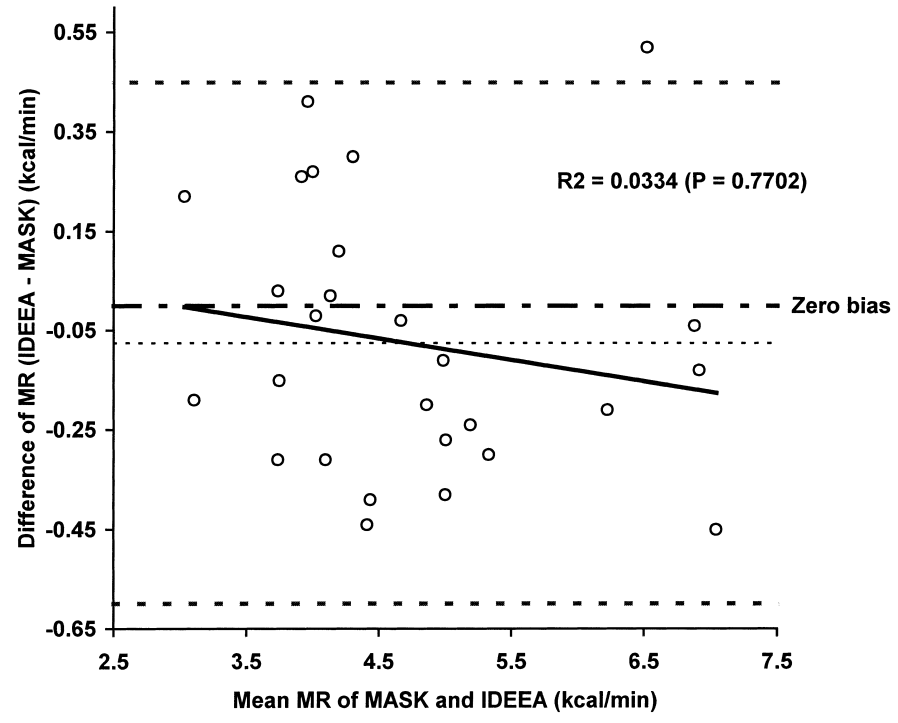

FIGURE 3-Difference of two methods against the average of two methods for the mask test.

database for transition. Figures 7 and 8 show that IDEEA underestimated MR during both the transition from standing to lying down and the transition from running to walking because the change of $\dot{\mathrm{VO}}_{2}$ is not a step response and decreases gradually because of the body buffering effect. By regression analysis, a second order polynomial was found to best fit MR changes during both transitions from running to walking (Fig. 7) and from standing to lying down (Fig. 8). Transition is part of PA, and is especially important for active persons who frequently change from one PA to another.

Unlike MR during normal sitting and standing, MR during fidgeting-like activities has not been well studied and documented. This could also contribute to the error in estimation. It has been demonstrated that MR during sitting while fidgeting could be 1.46 times the MR during motionless sitting, and MR during standing while fidgeting could be 1.69 times the MR during motionless standing (13). We believe that the underestimation of MR during fidgeting-like

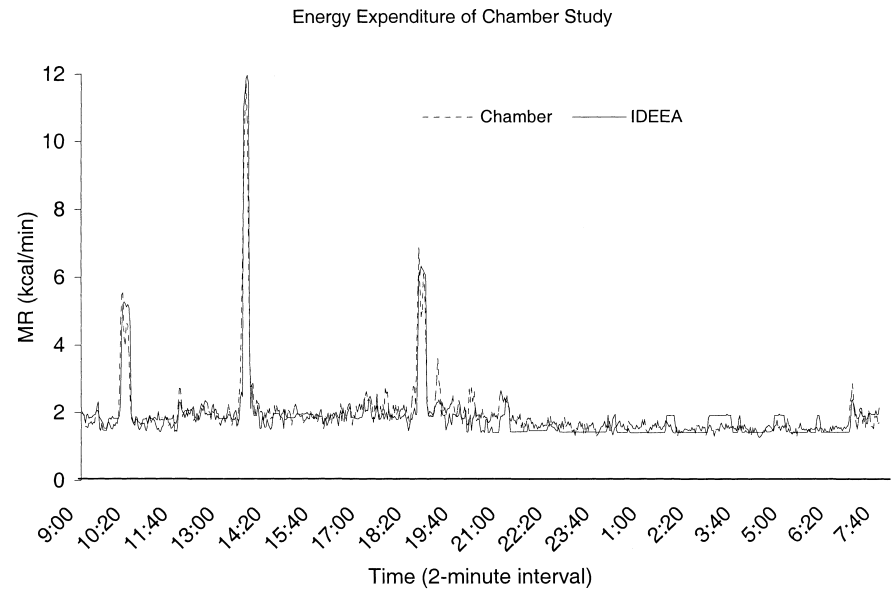

FIGURE 4-Representative profiles of 23-h MR estimated by IDEEA and 23-h MR measured by the chamber calorimeter. 


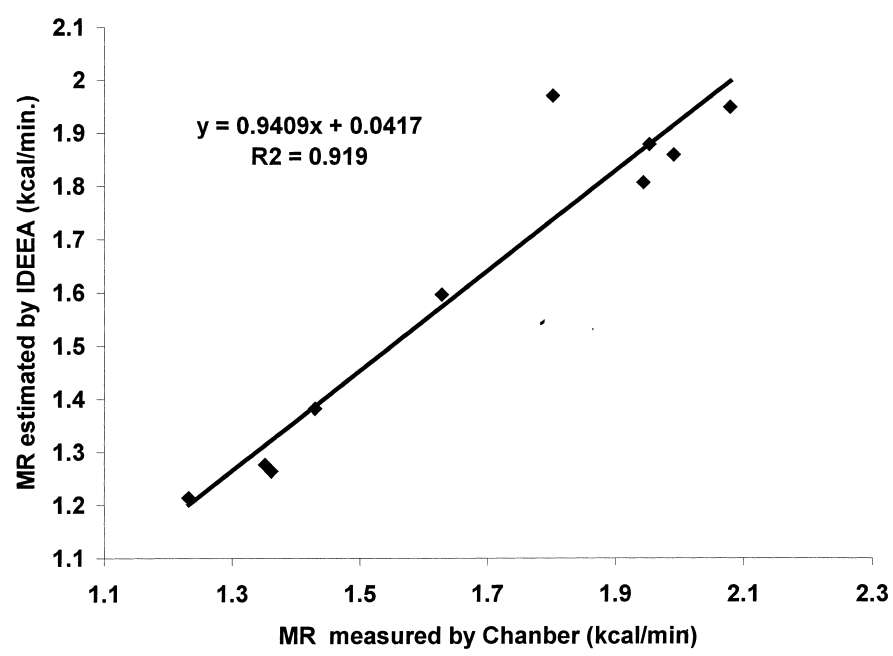

FIGURE 5-Comparison between MR from IDEEA and MR from the chamber calorimeter.

activities including the transition between activities is a major source of error, especially for chamber test during daytime, which should be investigated in the future.

For the chamber study, another source of error could be the assumption of a constant MR during sleep. We have previously shown that sleeping metabolic rate (SMR) is not constant and decreases with BMI during the sleep (29). Decrease of SMR and its relation to BMI:

$$
\text { slope }=-5 \times 10^{-6} \mathrm{BMI}^{2}+0.0002 \times \mathrm{BMI}-0.0024
$$

MR at the beginning of sleep can be as much as $12 \%$ higher than the average SMR and at the end of sleep can be as much as $12 \%$ lower than the average SMR depending on the slope of decrease of SMR (29). So, simply using one value of SMR without considering the slope of the decrease could contribute to the error. By applying above slope equation, the MR estimated from IDEEA was recalculated. Although overall accuracy was increased by only $1 \%$ from $95.2 \pm 2.3 \%$ to $96.2 \pm 1.9 \%$, a major improvement of SMR estimation was found (from $95.3 \pm 2.7 \%$ to $99.0 \pm 2.3 \%$,

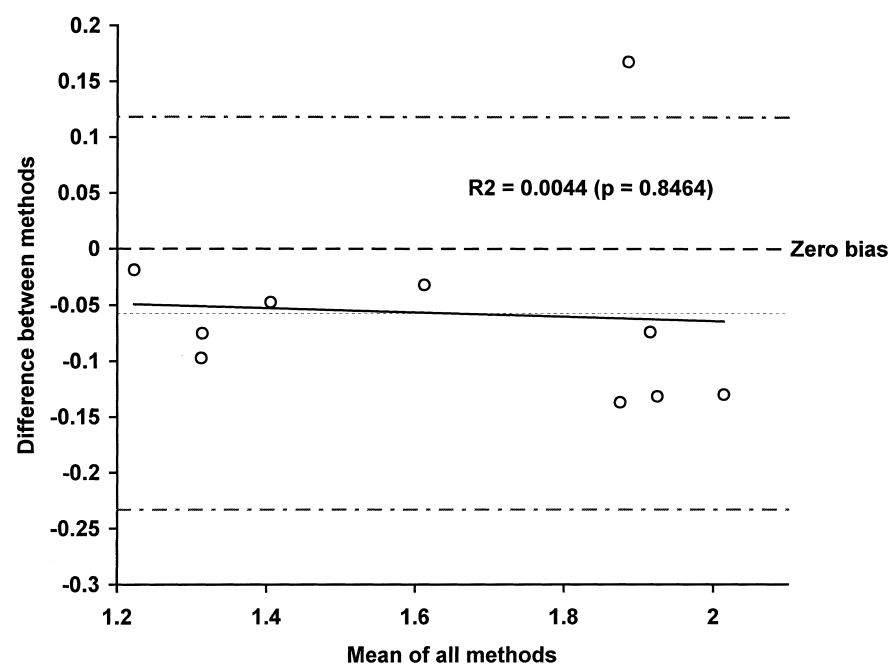

FIGURE 6-Difference of two methods against the average of two methods for chamber test.

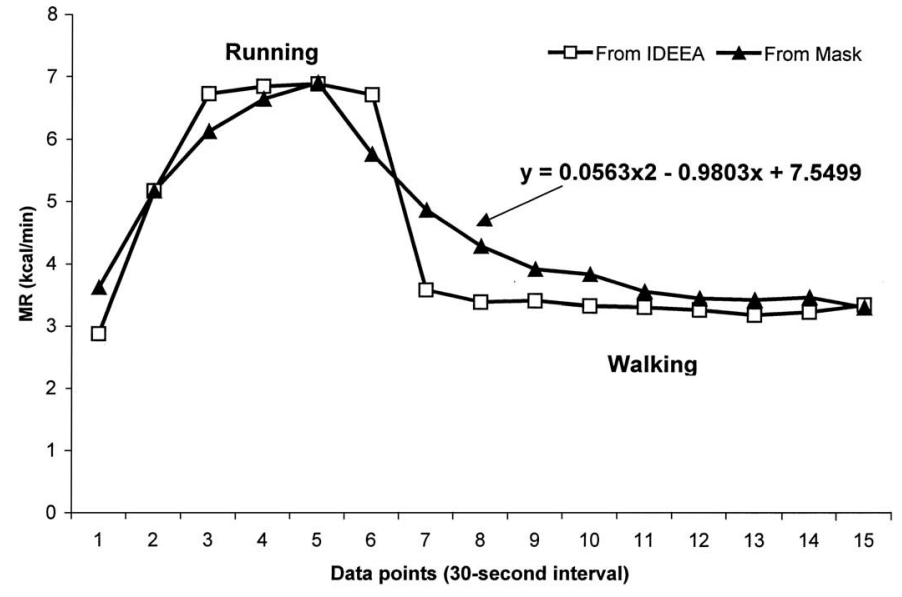

FIGURE 7-The MR from both IDEEA and the mask calorimeter during the transition from run to walk.

more than 4\%), proving that the slope of decrease of SMR is an important factor and should be considered in estimation of SMR.

The high accuracy of EE estimation by IDEEA is based on the accurate detection of type, onset, duration, and intensity of each PA, which we believe is the best way to tackle the measurement of daily PA. The operation is actually simple and easy, and takes only a few min before starting to collect data. Once the device is put on, the subject would not be distracted or interrupted until the device is taken off. This enables accurate recording of daily PA with minimal intrusion or interference. Note that at present, there are a few activities that cannot be performed with the device on, such as swimming and showering. No discomfort or inconvenience has been reported from more than 100 subjects who participated in our studies.

In summary, IDEEA provides a suitable alternative method for estimating energy expenditure with high accuracy. It provides both instantaneous and cumulative estimates of energy expenditure of physical activity over a given period.

This research was supported in part by NIH Grant NIDDK DK62152, P30KD2668, and DK07715.

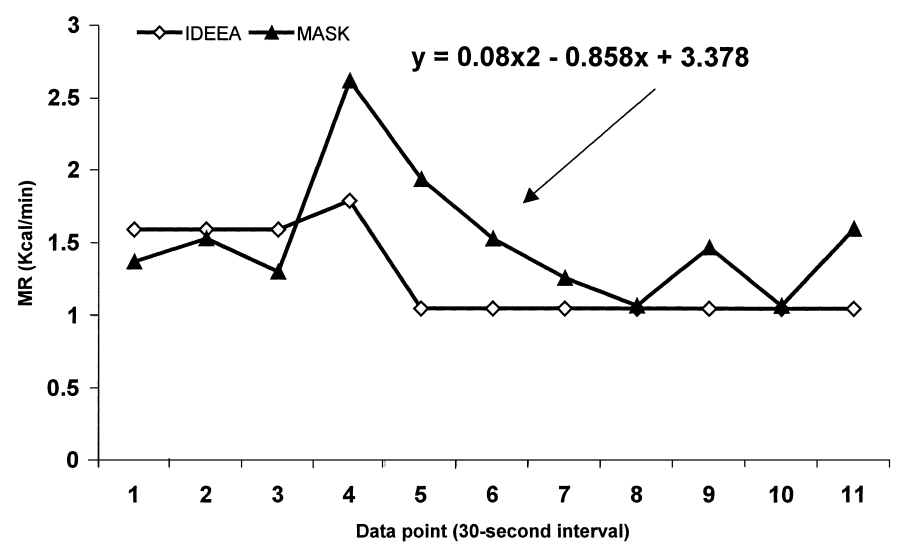

FIGURE 8-The MR from both IDEEA and the ask calorimeter during the transition between standing and lying down. 


\section{REFERENCES}

1. Ainsworth, B. E., D. R. Jacobs, JR., M. C. McNally, and A. A. LEON. Validity and reliability of self-reported physical activity status: the Lipid Research Clinics questionnaire. Med. Sci. Sports Exerc. 25:92-98, 1993.

2. Ainsworth, B. E., W. L. Haskell, A. S. Leon, et al. Compendium of Physical Activities: classification of energy costs of human activities. Med. Sci. Sports Exerc. 25:71-80, 1993.

3. Balogun, J. A., N. T. Farina, E. Fay, K. Rossmann, and L. Pozyc. Energy cost determination using a portable accelerometer. Phys. Ther. 66:1102-1109, 1986.

4. Blair, S. N., H. W. Kohl, N. F. Gordon and R. S. Paffenbarger. How much physical activity is good for health? Annu. Rev. Public Health 13:99-126, 1992.

5. Bouten, C. V., K. R. Westerterp, M. Verduin, and J. D. Janssen. Assessment of energy expenditure for physical activity using a triaxial accelerometer. Med. Sci. Sports Exerc. 26:1516-1523, 1994.

6. Bray, M. S., W. W. Wong, J. R. Morrow, N. F. Butte, and J. M. PIVARNIK. Caltrac versus calorimeter determination of 24-h energy expenditure in female children. Med. Sci. Sports Exerc. 26:15241530, 1994.

7. Cauley, J. A., R. E. LaPorte, R. Black-Sandler, M. M. SCHRAMM, and A. M. KRISKA. Comparison of methods to measure physical activity in postmenopausal women. Am. J. Clin. Nutr. 45:1422, 1987.

8. Eston, R. G., A. V. Rowlands, and D. K. Ingledew. Validity of heart rate, predometry, and accelerometry for predicting the energy cost of children's activities. J. Appl. Physiol. 84:362-371, 1998.

9. Gayle, R., H. J. Montoye, and J. Рhilpot. Accuracy of pedometers for measuring distance walked. Res. Q. 48:632-636, 1977.

10. Haymes, E. M., and W. C. Byrnes. Walking and running energy expenditure estimated by Caltrac and indirect calorimetry. Med. Sci. Sports Exerc. 25:1365-1369, 1993.

11. Hill, J. O., C. Melby, S. L. Johnson, and J. C. Peters. Physical activity and energy requirement. Am. J. Clin. Nutr. 62(Suppl.): 1059S-1066S, 1995.

12. Jakicic, J. M., C. Winters, K. Lagally, J. Ho, R. J. Roberson, and R. R. WING. The accuracy of the TriTrac-R3D accelerometer to estimate energy expenditure. Med. Sci. Sports Exerc. 31:747-754, 1999.

13. Levine, J. A., S. J. Schleusner, and M. D. Jensen. Energy expenditure of nonexercise activity. Am. J. Clin. Nutr. 72:1451-1454, 2000.

14. Luke, A., K. C. Maki, N. Barkey, R. Cooper, and D. McGee. Simultaneous monitoring of heart rate and motion to assess energy expenditure. Med. Sci. Sports Exerc. 29:144-148, 1997.

15. Meijer, G. A., K. R. Westerterp, H. Koper, and F. Ten Hoor. Assessment of energy expenditure by recording heart rate and body acceleration. Med. Sci. Sports Exerc. 21:343-347, 1989.
16. Montoye, H. J., R. Washburn, S. Servais, A. Ertl, J. G. Webster, and F. J. NAGLE. Estimation of energy expenditure by a portable accelerometer. Med. Sci. Sports Exerc. 15:403-407, 1983.

17. Pambianco, G., R. R. Wing, and R. Robertso. Accuracy and reliability of the Caltrac accelerometer for estimating energy expenditure. Med. Sci. Sports Exerc. 22:858-862, 1990.

18. Polls, M. A., P. H. Peters, H. B. Bueno De Mesquita, et al. Validity and repeatability of a modified Baecke questionnaire on physical activity. Intern. J. Epidemiol. 24:381-88, 1995.

19. Pugh, L. G. C. E. Oxygen intake in track and treadmill running with observation on the effect of air-resistance. J. Physiol. 207: 823-835, 1970.

20. Rising, R., I. T. Harper, A. M. Fontvielle, R. T. Ferraro, M. SPRAUl and E. Ravussin. Determinants of total daily energy expenditure: variability in physical activity. Am. J. Clin. Nutr. 59: 800-804, 1994.

21. Schoeller, D. A., and S. B. Racette. A review of field techniques for the assessment of energy expenditure. J. Nutr. 120:1492-1495, 1990.

22. Scholler, D. A. How accurate is self-reported dietary energy intake. Nutr. Rev. 48:373-79, 1990.

23. Schoeller, D. A. Measurement of energy expenditure in freeliving humans by using doubly labeled water. J. Nutr. 118:12781289, 1988.

24. Seale, J. L., and W. V. Rumpler. Comparison of energy expenditure measurement by diet records, energy intake balance, doubly labeled water and room calorimetry. Eur. J. Clin. Nutr. 51:856863, 1997.

25. Simons-Morton, B. G., and I. W. Huang. Heart rate monitor and Caltrac assessment of moderate-to-vigorous physical activity among preadolescent children (Abstract). Med. Sci. Sports Exerc. 23:S60, 1991.

26. Washburn, R. A., and H. J. Montoye. The assessment of physical activity by questionnaire. Am. J. Epidemiol. 125:563-576, 1986.

27. Washburn, R., M. K. Chin, and H. J. Montoye. Accuracy of pedometer in walking and running. Res. Q. Exerc. Sports 51:695702, 1980.

28. Weber, J. L., P. M. Reid, K. A. Greaves, et al. Original communication: validity of self-reported energy intake in lean and obese young women, using nutrient database, compared with total energy expenditure assessed by doubly labeled water. Eur. J. Clin. Nutr. 55:940-950, 2001.

29. Zhang, K., M. Sun, P. Werner, et al. Sleeping metabolic rate in relation to body mass index and body composition. Int. J. Obes. 3:376-383, 2002.

30. Zhang, K., P. Werner, M. Sun, F. X. Pi-Sunyer, and C. N. BoOzer. Measurement of daily physical activity. Obes. Res. 11: 33-40, 2003. 